\title{
QUADRATIC EXPRESSIONS IN A FREE BOSON FIELD $\left({ }^{1}\right)$
}

\author{
BY
}

ABEL KLEIN

\begin{abstract}
Quadratic expressions in a massive spinless free Boson field are treated by an appropriate extension of the method of second quantization. A certain class of these second quantized operators is shown to generate semigroups that act on a suitable scale of $L_{p}$-spaces, obtained through the diagonalization of the field at a fixed time, in a particularly regular fashion. The techniques are developed first in an abstract setting, and then applied to the neutral scalar free field. The locally correct generator of Lorentz transformations for $P(\varphi)_{2}$ is studied in detail, and essential selfadjointness is shown. These techniques are also used to solve explicitly the $\left(\varphi^{2}\right)_{n}$ model.
\end{abstract}

1. Introduction. We study quadratic expressions in a massive spinless free Boson field. Since their commutators with the field operators are linear expressions in the field, they can be treated by an appropriate extension of the method of second quantization. We introduce and study this extension, and as an application the locally correct generator of Lorentz transformations for $P(\varphi)_{2}$, introduced by Cannon and Jaffe [1], is worked out in detail, and selfadjointness is proved along the lines of the perturbation theory developed by Segal [11]. We also use these techniques to solve explicitly the $\left(\varphi^{2}\right)_{n}$ model.

We introduce the extension of the method of second quantization in an abstract setting, and study the regularity and covariance properties of the operators thus obtained ( $\$ 3$ ). An appropriate version (Theorem 1) of a theorem of Segal $[11$, Theorem 1] is then proved for a certain class of these operators $(\$ 4)$. The semigroups they generate are shown to act on a suitable scale of $L_{p}$-spaces, obtained through the diagonalization of the field at a fixed time, in a particularly regular fashion. The $L_{p}$-scale will depend on the particular operator, but the class of admissible perturbations will not only be independent of the operator, but will be the same one used by Segal [11].

We then turn to formal quadratic expressions in the neutral scalar free field, and show how they can be rigorously defined as second quantized operators $(\$ 5)$. The techniques developed are then used to study the locally correct generator of Lorentz transformations for $P(\varphi)_{2}(\$ 6)$. The free locally correct generator is a quadratic expression in the field operators, and is thus obtained as the second

Received by the editors March 31, 1972 and, in revised form, September 22, 1972. AMS (MOS) subject classifications (1970). Primary 81A81; Secondary 47-00, 47A99.

Key words and phrases. Locally correct generator of Lorentz transformations for $P(\varphi)_{2}$, free Weyl process, neutral scalar free field, unitarily implementable symplectic transformations.

(1) Supported by contract N00014-69-0200-4022.

Copyright $\odot 1973$, American Mathematical Society 
quantization of an operator of a type that can be skew-diagonalized (Theorem 2), and thus belongs to the class of operators to which Theorem 1 can be applied. As the interaction locally correct generator is of the same form as the interaction Hamiltonian, we obtain the essential selfadjointness of the total locally correct generator of Lorentz transformations.

Finally, we study the $\left(\varphi^{2}\right)_{n}$ model $(\S 7)$. We solve this model explicitly by performing a mass renormalization, for arbitrary space dimension. This has been done, using different techniques and formalism, by Ginibre and Velo [2]. (See also Rosen [6].) We explain the need for cutoffs, and show how they can be removed, leading to the renormalized Hamiltonian acting on the physical Hilbert space. This is done via a unitary renormalization of the cutoff Hamiltonian.

Segal has communicated to the author that he has a proof for the essential selfadjointness of the locally correct generator of Lorentz transformations, using his "calibrated theory" [12].

Part of the contents of this paper were contained in the author's Ph.D. thesis at the Massachusetts Institute of Technology. The author wishes to express his gratitude to Professor Irving Segal, for having introduced him to this research, and taken a continuing interest in its progress. The author is also in debt to Niels Skovhus Poulsen, Wilbert Wils, Michael Lennon and Barry Simon for helpful discussions. The author wants to thank Niels Skovhus Poulsen for permission to print his unpublished results.

2. Preliminaries. I. We shall use the convention that the inner product in a complex Hilbert space is antilinear in the first coordinate, and linear in the second.

II. If $\mathcal{H}$ is a given complex Hilbert space, the corresponding free Weyl process $(\mathcal{K}, W, \Gamma, v)$ consists of

(i) a complex Hilbert space $K$;

(ii) a continuous map $W$ from $\mathcal{H}$ to the unitary operators on $\mathcal{K}$ satisfying the Weyl relations:

$$
W(z) W\left(z^{\prime}\right)=\exp \left\{-(i / 2) \operatorname{Im}\left\langle z, z^{\prime}\right\rangle\right\} W\left(z+z^{\prime}\right), \text { for all } z, z^{\prime} \in \mathcal{H}
$$

(iii) a continuous unitary representation $U \rightarrow \Gamma(U)$ of the group of all unitary operators on $\mathcal{H}$, such that

(a) $\Gamma(U) W(z) \Gamma(U)^{-1}=W(U z)$ for all $U$ and $z$,

(b) $d \Gamma(A) \geq 0$ for any selfadjoint operator $A \geq 0$ in $\mathcal{H}$, where $d \Gamma(A)$

denotes the selfadjoint generator of the continuous unitary one-parameter group $\Gamma\left(e^{i t A}\right), t \in \mathbf{R}$;

(iv) a unit vector $v \in \mathcal{K}$ which is cyclic for the $W(z)$ and such that $\Gamma(U) v=$ $v$ for all $U$. 
The foregoing properties determine $(\mathcal{K}, W, \Gamma, v)$ uniquely, within unitary equivalence. The selfadjoint generator of the continuous unitary one-parameter group $W(t z), t \in \mathbf{R}$, will be denoted by $\Psi(z)$.

This definition is due to Segal.

III. For any operator $H$ in a real or complex Hilbert space, $D(H)$ will denote its domain.

3. Infinitesimal symplectics. Let $\mathcal{H}$ be a complex Hilbert space, and $(\mathcal{K}, W, \Gamma, v)$ the corresponding free Weyl process.

Definition 1. A symplectic transformation on $\mathcal{H}$ is a bounded invertible real linear operator $T$ such that $\operatorname{Im}\left\langle T z_{1}, T z_{2}\right\rangle=\operatorname{Im}\left\langle z_{1}, z_{2}\right\rangle$ for all $z_{1}, z_{2} \in \mathcal{H}$.

It is easy to verify that a bounded invertible real linear operator $T$ is a symplectic transformation if and only if $i T i^{-1}=\left(T^{t}\right)^{-1}$, where by $i$ we denote the operator multiplication by $i$ on $\mathcal{H}$, and $T^{t}$ is the transpose of $T$, i.e., $\operatorname{Re}\left\langle z_{1}, T z_{2}\right\rangle=$ $\operatorname{Re}\left\langle T^{t} z_{1}, z_{2}\right\rangle$ for all $z_{1}, z_{2} \in \mathcal{H}$.

Symplectic transformations come naturally into the context of quantum field theory. If $W$ is a Weyl map over $\mathcal{H}$, i.e., $W$ is a map from $\mathcal{H}$ into the unitary operators on a complex Hilbert space, such that $W(z) W\left(z^{\prime}\right)=\exp \left\{-(i / 2) \operatorname{Im}\left\langle z, z^{\prime}\right\rangle\right\}$. $W\left(z+z^{\prime}\right)$ for all $z, z^{\prime} \in \mathcal{H}$, it follows that if $T$ is a symplectic transformation on $\mathcal{H}, W \circ T$ is also a Weyl map.

Definition 2. A symplectic transformation $T$ on $\mathcal{H}$ is said to be unitarily implementable if there exists a unitary operator $U$ on $\mathcal{K}$ such that, for all $z \in \mathcal{H}$, $U W(z) U^{-1}=W(T z)$. We will write $\Gamma(T)$ for any such $U . \Gamma(T)$ is determined only up to a phase factor, which had been fixed for unitary operators $U$ by requiring $\Gamma(U) v=v$.

Shale [13] has studied the group of symplectic transformations, and has determined the class of unitarily implementable ones. $T$ is unitarily implementable if and only if $T^{t} T-I$ is a Hilbert-Schmidt operator (equivalent to $\left(T^{t} T\right)^{1 / 2}-I$ Hilbert-Schmidt).

Definition 3. A closed densely defined real linear operator $S$ in $\mathcal{H}$ is called an infinitesimal symplectic if it is the generator of a continuous one-parameter group of symplectic transformations $e^{t S}$. In case $S$ is the infinitesimal generator of a continuous one-parameter group, then $S$ is an infinitesimal symplectic if and only if $i S i^{-1}=-S^{t}$.

Definition 4. An infinitesimal symplectic $S$ is said to be selfadjointly implementable if there exists a selfadjoint operator $H$ in $K$ such that $e^{i t H}$ unitarily implements $e^{t S}$, for all $t \in \mathbf{R}$. We will write $H=\gamma(S)$. We note $H$ is determined only up to a scalar. If the vacuum $v$ is in the domain of $H$, we will fix $H$ by requiring $\langle v, H v\rangle=0$, and write $H=\gamma_{0}(S)$. 
Examples. 1. Let $A$ be a selfadjoint operator in $\mathcal{H}$. Then $i A$ is a selfadjointly implementable infinitesimal symplectic, and $\gamma_{0}(i A)=d \Gamma(A)$.

2. Let $K$ be a real linear self-transpose Hilbert-Schmidt operator on $\mathcal{H}$, such that $i K i^{-1}=-K$. Then $e^{t K}$ is a continuous one-parameter group of unitarily implementable symplectic transformations. By Theorem 4.2 of [13], there exists a continuous unitary one-parameter group $e^{i t H}$ implementing $e^{t K}$, as every continuous projective unitary representation of the real line can be made a bona fide representation. Thus $K$ is selfadjointly implementable. Actually, we can show more. $e^{i t H}$ can be exhibited explicitly, using the construction given in [13], and the vacuum can be shown to be in the domain of $H$ (see also Poulsen [4]).

3. Let $S$ be an infinitesimal symplectic, $T$ a symplectic transformation. Then $T S T^{-1}$ is again an infinitesimal symplectic. Moreover, if $S$ is selfadjointly implementable, and $T$ unitarily implementable, then the same is true of $T S T^{-1}$, and we can take $\gamma\left(T S T^{-1}\right)=\Gamma(T) \gamma(S) \Gamma(T)^{-1}$. One particularly interesting case is when $S=i A$, where $A$ is selfadjoint.

Remark l. Let $(\mathcal{H}, i,\langle\rangle$,$) be a complex Hilbert space, i.e., \mathcal{H}$ is a real vector space, $i$ is the operation of multiplication by $i$ that makes $\mathcal{H}$ into a complex vector space, $\langle$,$\rangle is the complex inner product. Let T$ be a symplectic transformation on $\mathcal{H}$. Then if $j=T^{-1} i T,\langle x, y\rangle_{T}=\langle T x, T y\rangle$ for $x, y \in \mathcal{H}$, $\left(\mathcal{H}, j,\langle,\rangle_{T}\right)$ is a complex Hilbert space, in which $\mathcal{H}$ is as before, $j$ gives the complex vector space structure, and $\langle,\rangle_{T}$ is the new inner product. Then if $S$ is a real linear operator on $\mathcal{H}$ such that $T S T^{-1}$ is complex linear in $(\mathcal{H}, i,\langle\rangle$,$) ,$ then $S$ is complex linear in $\left(\mathcal{H}, j,\langle,\rangle_{T}\right)$.

Proposition 1. Let $A$ and $B$ be selfadjointly implementable symplectics in $\mathcal{H}$, such that $(A+B)^{-}$generates a continuous one-parameter group. Then, if $\gamma(A)+\gamma(B)$ is essentially selfadjoint, $(A+B)^{-}$is selfadjointly implementable, and $\gamma\left((A+B)^{-}\right)=(\gamma(A)+\gamma(B))^{-}$.

Proof. By the Lie-Trötter formula

$$
e^{t(A+B)^{-}}=\operatorname{s-lim}_{n \rightarrow \infty}\left(e^{(t / n) A} e^{(t / n) B}\right)^{n}
$$

and

$$
e^{i t(\gamma(A)+\gamma(B))^{-}}=\operatorname{sim}_{n \rightarrow \infty}\left(e^{(i t / n) \gamma(A)} e^{(i t / n) \gamma(B)}\right)^{n} .
$$

Thus $(A+B)^{-}$is an infinitesimal symplectic, and for all $z \in \mathcal{H}$

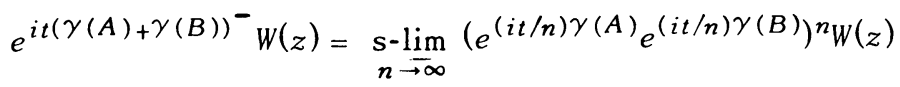

$$
\begin{aligned}
& =s-\lim _{n \rightarrow \infty} W\left(\left(e^{(t / n) A} e^{(t / n) B}\right)^{n} z\right)\left(e^{(i t / n) \gamma(A)} e^{(i t / n) \gamma(B)}\right)^{n}
\end{aligned}
$$

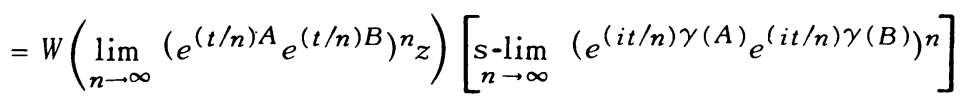


because both terms are bounded in norm, and $W$ is strongly continuous. But the last expression is just

$$
W\left(e^{t(A+B)^{-}} z\right) e^{i t(\gamma(A)+\gamma(B))^{-}}
$$

and thus $(\gamma(A)+\gamma(B))^{-}$selfadjointly implements $(A+B)^{-}$. End of proof.

Note. We have been somewhat careless in the proposition, as $\gamma(A), \gamma(B)$, and $\gamma\left((A+B)^{-}\right)$are defined only up to a constant. What we mean is that given a particular choice of $\gamma(A)$ and $\gamma(B),(\gamma(A)+\gamma(B))^{-}$is a possible choice for $\gamma\left((A+B)^{-}\right)$.

Particularly useful is the following result, due to N. Skovhus Poulsen [4].

Proposition 2 (Skovhus Poulsen). Let $A$ be selfadjoint in $\mathcal{H}, B$ bounded selfadjoint, $K$ real linear self-transpose Hilbert-Schmidt infinitesimal symplectic, $z \in \mathcal{H}$. Then the operators

$$
\gamma(i A)+\gamma(i B), \gamma(i A)+\gamma(K), \gamma(i A)+\Psi(z), \gamma(i A)+\gamma(K)+\Psi(z)
$$

are all essentially selfadjoint in any domain contained in the algebraic span of the $n$-particle subspaces $K_{n}$ in which $\gamma(i A)$ is essentially selfadjoint.

Example 4. Let $A$ be a selfadjoint operator in $\mathcal{H}, K$ a real linear HilbertSchmidt infinitesimal symplectic on $\mathcal{H}$. Then $i A+K$ is a selfadjointly implementable infinitesimal symplectic, and the vacuum is in the domain of $\gamma(i A+K)$. The statement follows from Propositions 1 and 2 , with the remark that $K=K_{1}+$ $K_{2}$, where $K_{1}=1 / 2\left(K-K^{t}\right), K_{2}=1 / 2\left(K+K^{t}\right)$, and $K_{1}$ is bounded and skew-transpose, which together with being an infinitesimal symplectic implies $K_{1}$ is complex linear, and thus skew-adjoint, and $K_{2}$ is a self-transpose Hilbert-Schmidt infinitesimal symplectic.

Remark 2. In [4], Skovhus Poulsen shows that if $S$ is a bounded selfadjointly implementable infinitesimal symplectic, then $S=i A+K$, where $A$ is bounded selfadjoint, and $K$ is Hilbert-Schmidt. It is natural to expect the more general selfadjointly implementable infinitesimal symplectic to be of the form $i A+K$, where $A$ is selfadjoint and $K$ is Hilbert-Schmidt, but this is just not true, as shown by the following counterexample.

Let $e_{a}$ be an orthonormal basis for $\mathcal{H}$, then $\mathcal{H}=\Sigma_{a} \oplus \mathcal{H}_{a}$ where $\mathcal{H}_{a}=\mathbf{C}$. for all $a$. Let $A$ be the selfadjoint operator given in $\mathcal{H}$ by $A=\Sigma_{a} \bigoplus a_{a} l_{\alpha}$, where $I_{a}$ is the identity operator in $\mathcal{H}_{a}$, and let $T$ be the symplectic transformation given by $T=\Sigma \bigoplus T_{a}$, where $T_{a}$ is represented by the matrix

$$
\left[\begin{array}{cc}
\lambda_{\alpha} & 0 \\
0 & \lambda_{\alpha}^{-1}
\end{array}\right]
$$


relative to the decomposition $\mathbf{C}=\mathbf{R} \oplus \mathbf{R}$, where $\lambda_{a}>0$, and $\Sigma_{\alpha}\left(\lambda_{a}-1\right)^{2}<\infty$. Then $T$ is unitarily implementable. Let $S=T i A T^{-1}$. Then $S$ is a selfadjointly implementable infinitesimal symplectic, and $S=\Sigma_{\alpha} \oplus S_{\alpha}$, where

$$
S_{a}=a_{\alpha}\left[\begin{array}{cc}
0 & -\lambda_{\alpha}^{2} \\
\lambda_{a}^{-2} & 0
\end{array}\right] \text {. }
$$

Thus, if we write $S=i B+K$, where $i B=1 / 2\left(S-S^{t}\right), K=1 / 2\left(S+S^{t}\right)$, then $B=$ $\Sigma_{\alpha} \oplus B_{\alpha}, B_{\alpha}=a_{\alpha}\left(\lambda_{\alpha}^{2}+\lambda_{\alpha}^{-2}\right) I_{\alpha}$, and so $B$ is selfadjoint, and

$$
K=\Sigma_{\alpha} \oplus K_{\alpha}
$$

where

$$
K_{\alpha}=a_{\alpha}\left[\begin{array}{cc}
0 & \lambda_{\alpha}^{-2}-\lambda_{\alpha}^{2} \\
\lambda_{a}^{-2}-\lambda_{\alpha}^{2} & 0
\end{array}\right],
$$

and so $K$ is a self-transpose infinitesimal symplectic. But $K$ is not necessarily Hilbert-Schmidt, we can choose $A$ and $T$ such that $K$ is unbounded. In fact, $K$ has discrete spectrum, and its eigenvalues are $\pm a_{\alpha}\left(\lambda_{\alpha}^{-2}-\lambda_{a}^{2}\right)$. We recall $\lambda_{a}=$ $1+t_{a}$, where $t_{a} \rightarrow 0$. Thus $\lambda_{a}^{-1} \approx 1-t_{\alpha}$, for a large enough, and $\left|\lambda_{a}^{-2}-\lambda_{a}^{2}\right| \approx$ $4\left|t_{a}\right| \cdot$. Thus, if we choose the $a_{a}^{\prime}$ 's and $\lambda_{a}^{\prime}$ 's such that $\left|a_{a}\left(\lambda_{a}-1\right)\right| \rightarrow \infty$, then $K$ is unbounded. In this case, we can also show that the vacuum is not in the domain of $\gamma(s)$, for an appropriate choice of the $a_{a}^{\prime}$ 's and $\lambda_{a}$ 's.

Remark 3. Another reasonable conjecture would be that if $S$ is a selfadjointly implementable infinitesimal symplectic, then $S=T i A T^{-1}$, for some unitarily implementable symplectic $T$ and selfadjoint $A$. But this is also not true. Recall that $S$ is of that form if and only if there exists a complex structure in $\mathcal{H}$ in which $S$ is skew-adjoint, or equivalently, in which $e^{t S}$ is a one-parameter group of unitary transformations. In [8] (see also Weinless [14]), Segal gives a simple criteria for that. $e^{t S}$ is unitarizable if and only if $\operatorname{Im}\left\langle x, e^{t S} y\right\rangle$ is a bounded function of $t$ for any $x, y \in \mathcal{H}$. So let $S=i A+K$, where $A$ is selfadjoint, and $K$ is a selftranspose Hilbert-Schmidt infinitesimal symplectic, and assume there exists $y \in$ $\mathcal{H}$ such that $A y=a y, K y=k y, a, k \neq 0$. Then $S$ is a selfadjointly implementable symplectic, but it cannot be of the form $T i A T^{-1}$, because for any $x \in \mathcal{H}$

$$
\operatorname{Im}\left\langle x, e^{t S} y\right\rangle=e^{i t a} e^{t k} \operatorname{Im}\langle x, y\rangle,
$$

and this is not bounded if $\operatorname{Im}\langle x, y\rangle \neq 0$.

The selfadjointly implementable infinitesimal symplectics $S$ of the form 
$T i A T^{-1}$ or $i A+K, K$ Hilbert-Schmidt, are easier to deal with than the general case. In these cases, in addition to $e^{i t \gamma(S)} W(z) e^{-i t \gamma(S)}=W\left(e^{t S} z\right)$, we can also prove

Proposition 3. Let $S$ be of the form $T_{i A T^{-1}}$ or $i A+K$, where $A$ is selfadjoint, $T$ symplectic, $K$ Hilbert-Scbmidt infinitesimal symplectic. Then, if $H=\gamma(S)$,

$$
e^{-i \boldsymbol{\Psi}(z)} H e^{i \boldsymbol{\Psi}(z)}=(H+\Psi(S z)+1 / 2 \operatorname{Im}\langle z, S z\rangle)^{-}
$$

for all $z \in D(S)$.

Proof. For $S=T i A T^{-1}$, it follows from Lemma 3.3 of Segal [9]. For $S=$ $i A+K$, we first notice that the left-hand side is essentially selfadjoint on $D(\gamma(i A))$ intersected with the algebraic span of the $n$-particles subspaces $\mathcal{K}_{n}$, by Proposition 2, and thus it is enough to show the formula holds in this domain. With that, the proof is the same as that of Lemma 3.3 of [9], except that one should use

$$
\left.e^{-i \boldsymbol{\Psi}(z)} e^{i t H} e^{i \boldsymbol{\Psi}(z)}=e^{i t H} e^{-i \boldsymbol{\Psi}(z}-t^{\prime}\right) e^{i \boldsymbol{\Psi}(z)},
$$

where $z_{-t}=e^{-t S} z$, instead of the similar formula used there. End.

4. Regularity of the action on an appropriate $L_{p}$-scale. In [11], Segal introduced a perturbation theory appropriate to quantum processes, based on the action of the semigroups $e^{-t H}$ on a certain scale of $L_{p}$-spaces, where $H=d \Gamma(A)$, and $A$ is a strictly positive selfadjoint operator, leaving $\mathcal{H}^{\prime}$ invariant, where $\mathcal{H}^{\prime}$ is a real Hilbert space of which $\mathcal{H}$ is the complexification. We extend these results for infinitesimal symplectics.

Let $\mathcal{H}$ denote the complexification of the real Hilbert space $\mathcal{H}^{\prime}$, and let $(K, W, \Gamma, v)$ be the corresponding free Weyl process. Let $A$ be any given selfadjoint operator in $\mathcal{H}^{\prime}$ such that $A \geq \epsilon>0$, and let us also denote by $A$ its extension to $\mathcal{H}$ as a complex linear selfadjoint operator. Let $H_{0}=d \Gamma(A)$. Let $R$ be a bounded linear operator on $\mathcal{H}^{\prime}$, with bounded inverse, and such that $R^{t} R-I$ is Hilbert-Schmidt. Let $T=R \oplus\left(R^{t}\right)^{-1}$, corresponding to $\mathcal{H}=\mathcal{H}^{\prime} \oplus \mathcal{H}^{\prime}$. Then $T$ is a unitarily implementable symplectic transformation in $\mathcal{H}$. Let $H=$ $\Gamma(T) H_{0} \Gamma(T)^{-1}$.

Theorem 1. There exists a weak probability measure $\mu$ on $\mathcal{H}^{\prime}$, depending only on $|T|=\left(T^{t} T\right)^{1 / 2}$, such that $\mathcal{K}$ can be identified with $L_{2}\left(\mathcal{H}^{\prime}, d \mu\right)$, and

(i) $e^{-t H}$ is a contraction in $L_{p}\left(\mathcal{H}^{\prime}, d \mu\right)$ for all $t \geq 0$ and $1 \leq p \leq \infty$;

(ii) for all $p \geq 2$ there exists $t_{0}(p) \geq 0$ such that, for $t \geq t_{0}(p), e^{-t H}$ is a contraction from $L_{2}\left(\mathcal{H}^{\prime}, d \mu\right)$ to $L_{p}\left(\mathcal{H}^{\prime}, d \mu\right)$; 
(iii) if $g$ is the isonormal weak probability measure on $\mathcal{H}^{\prime}$,

$$
\bigcap_{p \in[1, \infty)} L_{p}\left(\mathcal{H}^{\prime}, d g\right)=\bigcap_{p \in[1, \infty)} L_{p}\left(\mathcal{H}^{\prime}, d \mu\right) .
$$

Proof. We can assume $T=|T|$, i.e., $R=|R|$, because $T=|T| O$, where $O$ is an orthogonal symplectic, hence unitary, and thus $\Gamma(O) d \Gamma(A) \Gamma(O)^{-1}$ is of the form $d \Gamma\left(A^{\prime}\right)$, where $A^{\prime}=O A O^{-1}$ has the same properties as $A$.

We will use the real wave representation for $(\mathcal{K}, W, \Gamma, v)$ (see [11], [7]). In this representation $\mathcal{K}=L_{2}\left(\mathcal{H}^{\prime}, d g\right)$, where $g$ is the isonormal weak probability measure, and $v$ is the function 1 . We recall ([7], [13]) that in this representation the tame functions over $\mathcal{H}^{\prime}$ form a dense set in $\mathcal{K}$, and we can choose $\Gamma(T)$ such that

$$
(\Gamma(T) f)(x)=f(R x) w(x) \text {. for all tame functions } f
$$

where $w=\sqrt{d \mu / d g}$, and $\mu$ is the weak probability measure on $\mathcal{H}^{\prime}$ defined by

$$
\int f(x) d \mu(x)=\int f\left(R^{-1} x\right) d g(x)
$$

In particular, $\Gamma(T) v=w$. Let $Z$ be the unitary operator

$$
Z: L_{2}\left(\mathfrak{H}^{\prime}, d g\right) \rightarrow L_{2}\left(\mathfrak{H}^{\prime}, d \mu\right), \quad f \rightarrow f w^{-1} .
$$

Then $Z w=1$, and we can identify $\mathcal{K}$ with $L_{2}\left(\mathcal{H}^{\prime}, d \mu\right)$.

Lemma 1. Let $Y=Z \Gamma(T)$. Then $Y$ is a Banach space isomorphism from $L_{p}\left(\mathcal{H}^{\prime}, d g\right)$ to $L_{p}\left(\mathcal{H}^{\prime}, d \mu\right)$ for all $p \in[1, \infty]$.

Proof. $(Z \Gamma(T) f)(x)=f(R x)$, so

$$
\int|Y f(x)|^{p} d \mu(x)=\int|f(R x)|^{p} d \mu(x)=\int|f(x)|^{p} d g(x) .
$$

End.

For an operator $X$ in $\mathcal{K}$, let us denote by $\tilde{X}$ its representation as an operator in $L_{2}\left(\mathcal{H}^{\prime}, d \mu\right)$, i.e. $\widetilde{X}=Z X Z^{-1}$. Then $\widetilde{H}=Z \Gamma(T) H_{0} \Gamma(T)^{-1} Z^{-1}=Y H_{0} Y_{-t H_{0}}^{-1}$. Thus $e^{-t H}=Y e^{-t H_{0}} Y^{-1}$, so $e^{-t H}$ acts on $L_{p}\left(\mathcal{H}^{\prime}, d \mu\right)$ in the same way $e^{-t H_{0}}$ acts on $L_{p}\left(\mathcal{H}^{\prime}, d g\right)$, so (i), (ii) follow from Theorem 1 of [11].

(iii) follows by Hölder's inequality, from the following lemma.

Lemma 2. There exists $p, q>1$ such that $w^{2} \in L_{p}\left(\mathcal{H}^{\prime}, d g\right)$ and $w^{-2} \epsilon$ $L_{q}\left(\mathcal{H}^{\prime}, d \mu\right)$.

Proof. Let $\left\{e_{i}\right\}$ be an orthonormal basis for $\mathcal{H}^{\prime}$, such that $R e_{i}=c_{i} e_{i}$, where $0<c_{i}=1+\lambda_{i}, \Sigma \lambda_{i}^{2}<\infty$. Such a basis exists, as $R-I$ is Hilbert-Schmidt. Let 
$(\mathcal{K}, W, \Gamma, v)$ be represented as in Corollary 3.6 of $[7]$. Then $\mathcal{K}$ is represented by $L_{2}(M)$, where $M=(\mathbf{R}, d g)^{\operatorname{dim} \mathcal{H}^{\prime}}$, and $L_{p}\left(\mathcal{H}^{\prime}, d g\right)=L_{p}(M)$.

Let $w_{i}(x)=\sqrt{c_{i}} \exp \left\{-1 / 4\left(c_{i}^{2}-1\right) x^{2}\right\} \in L_{2}(\mathbf{R}, d g)$. Then $w(x)=\bigoplus_{i} w_{i}(x)$. $\mathbb{W e}_{2}$ claim there exists $p>1$ such that $w_{i}^{2}(x) \in L_{p}(\mathbf{R}, d g)$ for all $i$, and $\left\|w_{i}^{2}\right\|_{p} \leq e^{\eta \lambda_{i}^{2}}$, where $\eta$ is a constant independent of $i$. In fact,

$$
\begin{aligned}
\left\|w_{i}^{2}\right\|_{p} & =c_{i}\left(\int \exp \left\{-\frac{p}{2}\left(c_{i}^{2}-1\right) x^{2}\right\} \exp \left\{-\frac{1}{2} x^{2}\right\} \frac{d x}{(2 \pi)^{1 / 2}}\right)^{1 / p} . \\
& =c_{i}\left(\int \exp \left\{-\frac{1}{2}\left(p\left(c_{i}^{2}-1\right)+1\right) x^{2}\right\} \frac{d x}{(2 \pi)^{1 / 2}}\right)^{1 / p} .
\end{aligned}
$$

For this to be finite we need $1+p\left(c_{i}^{2}-1\right) \geq 0$. We have two cases:

(a) $c_{i} \geq 1$. Then this is true for all $p$.

(b) $c_{i}<1$. Then we want $p \leq 1 /\left(1-c_{i}^{2}\right)$.

We notice $1 /\left(1-c_{i}^{2}\right)>1$, hence if $c_{0}=\min c_{i}$, then $1 /\left(1-c_{i}^{2}\right) \geq 1 /\left(1-c_{0}^{2}\right)>1$, as $c_{0}>0$ follows from the compactness of $R-I$. Thus if we choose $1<p<$ $1 /\left(1-c_{0}^{2}\right),\left\|w_{i}^{2}\right\|_{p}<\infty$ for all $i$. Moreover

$\left\|w_{i}^{2}\right\|_{p}=c_{i}\left(1+p\left(c_{i}^{2}-1\right)\right)^{-1 / 2 p}=\left(\frac{c_{i}^{2 p}}{1+p\left(c_{i}^{2}-1\right)}\right)^{1 / 2 p}=\left(\frac{\left(1+\lambda_{i}\right)^{2 p}}{1+p\left(\left(1+\lambda_{i}\right)^{2}-1\right)}\right)^{1 / 2 p}$.

Let $f(\lambda)=(1+\lambda)^{2 p} /\left(1+p\left((1+\lambda)^{2}-1\right)\right)$. Then, by Taylor's formula, $f(\lambda)=f(0)$ $+f^{\prime}(0) \lambda+1 / 2 f^{\prime \prime}\left(\lambda_{1}\right) \lambda^{2}$, where $0 \leq \lambda_{1} \leq \lambda$ if $\lambda>0$, or $\lambda \leq \lambda_{1} \leq 0, \lambda<0$.

But $f(0)=1, f^{\prime}(0)=0$, and $f^{\prime \prime}(\lambda)$ is a continuous function of $\lambda$ and thus there exists $\eta^{\prime}$ such that $\left|f^{\prime \prime}(\lambda)\right| \leq 2 \eta^{\prime}$ for $\lambda \in\left[\min \lambda_{i}\right.$, max $\left.\lambda_{i}\right]$. Hence $|f(\lambda)| \leq$ $1+\eta^{\prime} \lambda^{2} \leq e^{\eta^{\prime} \lambda^{2}}$ for $\lambda$ in this interval. Therefore

$$
\left\|w_{i}^{2}\right\|_{p} \leq e^{\eta^{\prime} \boldsymbol{\lambda}_{i}^{2} / 2 p}=e^{\eta \boldsymbol{\lambda}_{i}^{2}} \text { for } \eta=\eta^{\prime} / 2 p
$$

Thus $\left\|w^{2}\right\|_{p} \leq \exp \left(\eta\left(\Sigma_{i} \lambda_{i}^{2}\right)\right)<\infty$, so $w^{2} \in L_{p}\left(\mathcal{H}^{\prime}, d g\right)$ for some $p>1$.

Similarly, we can show $w^{-2} \in L_{r}\left(\mathcal{H}^{\prime}, d g\right)$ for some $r>0$, and this implies $w^{-2} \in L_{r+1}\left(\mathcal{H}^{\prime}, d \mu\right)$. End of proof.

Remark 4. (iii) says that the class of admissible (for Theorem 2 of [11]) perturbations for $H$ as in the theorem is independent of $H$, and is the same as the one used by Segal [11].

Remark 5. It follows from the proof that $e^{-t H}$ has a positive kernel (i.e., $\left\langle f, e^{-t H} g\right\rangle \geq 0$ if $\left.f, g \geq 0\right)$ in both $L_{2}\left(\mathcal{H}^{\prime}, d g\right)$ and $L_{2}\left(\mathcal{H}^{\prime}, d \mu\right)$.

Remark 6. Let $\mathcal{H}_{T}=\left(\mathcal{H}, T i T^{-1},\langle,\rangle_{T^{-1}}\right)$ (see Remark 1). Then $T: \mathcal{H} \rightarrow$ $\mathcal{H}_{T}$ is a unitary map, $T \mathcal{H}^{\prime}=\mathcal{H}^{\prime}$, and $\mathcal{H}_{T}=\mathcal{H}^{\prime}+T i T^{-1} \mathcal{H}^{\prime}$. Moreover, $\mu$ is the 
isonormal weak probability measure over the real Hilbert space $\mathcal{H}_{T}^{\prime}=\left(\mathcal{H}^{\prime},\langle,\rangle_{T^{-1}}\right)$. If $\left(\mathcal{K}_{T}, W_{T}, \Gamma_{T}, v_{T}\right)$ is the real wave representation for the free Weyl process over $\mathcal{H}_{T}$, then $\mathcal{K}_{T}=L_{2}\left(\mathcal{H}^{\prime}, d \mu\right)$ and $Y: L_{2}\left(\mathcal{H}^{\prime}, d g\right) \rightarrow L_{2}\left(\mathcal{H}^{\prime}, d \mu\right)$ intertwines $(\mathcal{K}, W, \Gamma, v)$ and $\left(\mathcal{K}_{T}, W_{T}, \Gamma_{T}, v_{T}\right)$ unitarily implementing $T: \mathcal{H} \rightarrow \mathcal{H}_{T}$, i.e., $W_{T}(z)=Y W\left(T^{-1} z\right) Y^{-1}$ for $z \in \mathcal{H}^{\prime}, \Gamma_{T}(U)=Y \Gamma\left(T^{-1} U T\right) Y^{-1}$ for $U$ unitary on $\mathcal{H}_{T}$, and $v_{T}=Y v$.

$T i A T^{-1}$ is a skew-adjoint operator in $\mathcal{H}_{T}$, say $=T i T^{-1} B, B$ selfadjoint in $\mathcal{H}_{T}$. Thus

$$
d \Gamma_{T}(B)=Y d \Gamma(A) Y^{-1}=Y H_{0} Y^{-1}=\tilde{H} .
$$

It follows (i), (ii) hold as $\widetilde{H}=d \Gamma_{T}(B)$, and to prove (iii) it would have been enough to show

$$
\bigcap_{p \in[1, \infty)} L_{p}\left(\mathcal{H}^{\prime}, d g\right) \subset \bigcap_{p \in[1, \infty)} L_{p}\left(\mathcal{H}^{\prime}, d \mu\right)
$$

as we can invert the roles of $g$ and $\mu$.

Corollary. In the case of the neutral scalar free field with mass $m>0$, in two dimensional space-time, if $H$ is as in the theorem, $P$ is any given real polynomial bounded from below, and $V(f)$ is the selfadjoint operator represented by $\int: P(\varphi(x)): f(x) d x$, where $f \in L_{1} \cap L_{2}(\mathbf{R}), f \geq 0, H+V(f)$ is essentially selfadjoint, and bounded from below.

Proof. Follows from the theorem, and from Theorem 2 and proof of Corollary 3.3 of [11]. End.

5. Quadratic expressions in the neutral scalar free field and its derivatives. Let $\mathcal{H}=L_{2}\left(\mathbf{R}^{n}\right), m>0$. If $C$ denotes the operator $\left(m^{2}-\Delta\right)^{1 / 4}$ in $\mathcal{H}$, then, within unitary equivalence, the neutral scalar free field $\phi$ with mass $m$, and its time derivative $\dot{\phi}$, are given, at time zero, by

$$
\phi(f)=\phi(f, 0)=\Psi\left(C^{-1} f\right), \quad \phi(g)=\dot{\phi}(g, 0)=\Psi(i C g),
$$

for real functions $f \in L_{2}\left(\mathbf{R}^{n}\right), g \in D(C)$. We then have

$$
[\phi(f), \dot{\phi}(g)]^{-}=i\langle f, g\rangle, \quad\left[\phi\left(f_{1}\right), \phi\left(f_{2}\right)\right]^{-}=0=\left[\dot{\phi}\left(g_{1}\right), \dot{\phi}\left(g_{2}\right)\right]^{-},
$$

or, symbolically, if $\phi(f) \sim \int \varphi(x) f(x) d x$, and $\dot{\phi}(g) \sim \int \dot{\varphi}(y) g(y) d y,[\varphi(x), \dot{\varphi}(y)]=$ $i \delta(x-y),[\varphi(x), \varphi(y)]=0=[\dot{\varphi}(x), \dot{\varphi}(y)]$. Time evolution is given by the free Hamiltonian $H_{0}=d \Gamma\left(C^{2}\right)$.

Let 2 be a real quadratic expression in $\varphi(x), \dot{\varphi}(x)$ and its derivatives, e.g. $Q(x)=m^{2} \boldsymbol{\varphi}(x)^{2}+\nabla \boldsymbol{\varphi}(x)^{2}+\dot{\varphi}(x)^{2}$. Let us consider the formal expression $H=\int: Q(x): f(x) d x$, where $f$ is some real function. We want to make sense out of $H$. All we really know about $H$ are its commutation relations with $\phi$ and $\dot{\phi}$, 
or equivalently, its commutation relations with $\Psi . Q$ being a real quadratic expression, $i[H, \Psi(z)]=\Psi(S z)$, where $S$ is a real linear operator in $\mathcal{H}$, and $S$ must be an infinitesimal symplectic, because

$$
\begin{aligned}
\Psi\left(i S i^{-1} z\right) & =\Gamma(i) \Psi\left(S^{-1} z\right) \Gamma(i)^{-1}=\Gamma(i) i\left[H, \Psi\left(i^{-1} z\right)\right] \Gamma(i)^{-1} \\
& =i\left[\Gamma(i) H \Gamma(i)^{-1}, \Psi(z)\right]=\Psi\left(-S^{t} z\right)
\end{aligned}
$$

as $\Gamma(i) \Psi(z) \Gamma\left(i^{-1}\right)=\Psi(i z)$. We know that if $S$ is selfadjointly implemented by $\gamma(S)$, then $i[\gamma(S), \Psi(z)]=\Psi(S z)$, for $z$ in the domain of $S$, as $e^{i t \gamma(S)} W(z) e^{-i t \gamma(S)}=$ $W\left(e^{t S} z\right)$. If moreover we require $H$ to have zero vacuum expectation, which is equivalent to Wick ordering the terms in the quadratic expression $Q$, we fix $\gamma(S)$ by requiring $\langle v, \gamma(S) v\rangle=0$. We are thus led to define $H$ by $\gamma_{0}(s)$, and thus obtain $H$ as a selfadjoint operator.

6. The locally correct generat or of Lorentz transformations for $P(\varphi)_{2}$. The locally correct generator of Lorentz transformations,

$$
M\left(g_{1}, g_{2}\right)=\epsilon H_{0}+M_{0}\left(g_{1}\right)+M_{I}\left(g_{2}\right)
$$

where

$$
\begin{aligned}
& M_{0}\left(g_{1}\right)=\frac{1}{2} \int: m^{2} \boldsymbol{\varphi}(x)^{2}+\nabla_{\boldsymbol{\varphi}}(x)^{2}+\dot{\varphi}(x)^{2}: g_{1}(x) d x, \\
& M_{I}\left(g_{2}\right)=\int: P(\boldsymbol{\varphi}(x)): g_{2}(x) d x,
\end{aligned}
$$

$\epsilon>0, g_{1}, g_{2} \in \mathcal{S}(\mathbf{R}), g_{1}, g_{2} \geq 0, P$ a polynomial bounded from below, has been introduced and studied by Cannon and Jaffe [1]. They treated the $\left(\varphi^{4}\right)_{2}$ case, and their results have been extended to $\left(\varphi^{2 n}\right)_{2}$ by Rosen [5].

Notice that $\epsilon H_{0}+M_{0}\left(g_{1}\right)=M_{0}(f)$, where $f=\epsilon+g_{1} . M_{0}(f)$ is a real quadratic expression in the field and its derivatives, so we apply to it the procedure outlined in the last section.

Formally, $\left[M_{0}(f), \phi(b)\right]=-i \phi\left(M_{f} b\right),\left[M_{0}(f), \dot{\phi}(b)\right]=i \phi\left(\left(m^{2} M_{f}-\nabla M_{f} \nabla\right) b\right)$, where $M_{f}$ denotes multiplication by $f$, and $\nabla=d / d x$. Recalling $\phi(b)=\Psi\left(C^{-1} b\right)$, $\dot{\phi}(b)=\psi(i C b)$, we have $i\left[M_{0}(f), \Psi(z)\right]=\Psi(S z)$, where $S$ has the matrix representation

$$
\left[\begin{array}{cc}
0 & -C^{-1}\left(m^{2} M_{f}-\nabla M_{f} \nabla\right) C^{-1} \\
C M_{f} C & 0
\end{array}\right]
$$

if we write $L_{2}(\mathbf{R})=R L_{2}(\mathbf{R}) \oplus R L_{2}(\mathbf{R})$, where $R L_{2}$ is the real $L_{2}$.

Thus, $S=i A+K$, where 


$$
\begin{array}{ll}
A=\frac{1}{2}\left[\begin{array}{ll}
L & 0 \\
0 & L
\end{array}\right], \quad L=C M_{f} C+C^{-1}\left(m^{2} M_{f}-\nabla M_{f} \nabla\right) C^{-1}, \\
K=\frac{1}{2}\left[\begin{array}{ll}
0 & Y \\
Y & 0
\end{array}\right], \quad Y=C M_{f} C-C^{-1}\left(m^{2} M_{f}-\nabla M_{f} \nabla\right) C^{-1} .
\end{array}
$$

We assume $f=\epsilon+g_{1}, \epsilon \geq 0,0 \leq g_{1} \in \mathcal{S}(\mathbf{R})$. It follows $Y$ is a Hilbert-Schmidt operator, because in Fourier transform space it is an integral operator with kernel

$$
Y(k, p)=\frac{\left(m^{2}+k^{2}\right)^{1 / 2}\left(m^{2}+p^{2}\right)^{1 / 2}-m^{2}-k p}{\left(m^{2}+k^{2}\right)^{1 / 4}\left(m^{2}+p^{2}\right)^{1 / 4}} \hat{g}_{1}(k-p),
$$

where $\hat{g}_{1}(k)=\int e^{i k x} g_{1}(x) d x$, and $Y(k, p)$ is an $L_{2}$-kernel by Lemma 3.2.2 of [1]. Thus $K$ is a real linear self-transpose Hilbert-Schmidt infinitesimal symplectic. In case $\epsilon>0, M_{f}$ has a bounded inverse, and so $C M_{f} C$ is a real selfadjoint operator, being the inverse of the bounded real selfadjoint operator $C^{-1} M_{f}^{-1} C^{-1}$. If $\epsilon=0, C M_{f} C \geq 0$, and we can take its Friedrichs' extension. In either case we obtain $L$ as a real selfadjoint operator, and thus $A$ as a selfadjoint operator.

It follows (see $\$ 3$ ) that $S$ is selfadjointly implementable, and $v \in D(\gamma(S)$ ). We thus define $M_{0}(f)$ rigorously by $M_{0}(f)=\gamma_{0}(s)$, as $\gamma_{0}(s)$ is a selfadjoint operator having the required commutation relations and zero vacuum expectation. It also follows that $M_{0}(f)$ is essentially selfadjoint on any core for $d \Gamma(A)$ contained in the algebraic span of the $n$-particles subspaces.

In particular, if $\epsilon=0, M_{0}\left(g_{1}\right)$ is still defined as a selfadjoint operator. We would like to notice that John Cannon has a nonpublished proof for that (private communication).

We now want to show that $M\left(g_{1}, g_{2}\right)=M_{0}(f)+M_{I}\left(g_{2}\right)$, with $f=\epsilon+g_{1}, \epsilon>0$, $0 \leq g_{1}, g_{2} \in \mathscr{S}(\mathbf{R})$, is essentially selfadjoint. By the Corollary to Theorem 1 , it suffices to show that $M_{0}(f)$ satisfies the hypothesis of Theorem 1 .

This will follow from

Theorem 2. Let $\mathcal{H}$ be a complex Hilbert space, and $\mathcal{H}$ ' a real subspace. such that $\mathcal{H}=\mathcal{H}^{\prime}+i \mathcal{H}^{\prime}$. Let

$$
S=\left[\begin{array}{cc}
0 & -\alpha^{2} \\
\beta^{2} & 0
\end{array}\right]
$$

where $\alpha^{2}, \beta^{2} \geq \epsilon>0$ are selfadjoint operators in $\mathcal{H}^{\prime}$, sucb that $\beta^{2}-\alpha^{2}$ is Hilbert-Schmidt (or bounded, or trace class), and $\alpha\left(\beta^{2}-\alpha^{2}\right)$ is densely defined. 
Then $R=\left(\alpha \beta^{2} \alpha\right)^{1 / 4} \alpha^{-1}$ is a bounded invertible operator in $\mathcal{H}^{\prime}$, with a bounded inverse, such that $R^{t} R-I$ is Hilbert-Schmidt (or bounded, or trace class).

If $T=R \oplus\left(R^{t}\right)^{-1}, T S T^{-1}=i\left(\alpha \beta^{2} \alpha\right)^{1 / 2}$, where $\left(\alpha \beta^{2} \alpha\right)^{1 / 2}$ is selfadjoint and $\geq \epsilon>0$.

Corollary. The locally correct generator of Lorentz transformations for $P(\varphi)_{2}$ is essentially selfadjoint and bounded from below.

Proof of Corollary. $M_{0}(f)=\gamma_{0}(S)$, where $S$ has the form

$$
\left[\begin{array}{cc}
0 & -(L-Y) \\
L+Y & 0
\end{array}\right],
$$

where $L$ and $Y$ are selfadjoint operators in $\mathcal{H}^{\prime}, Y$ is Hilbert-Schmidt, and it is easy to check that $L-Y \geq \epsilon>0, L+Y \geq \epsilon>0$. As $R \mathscr{S}(\mathbf{R})$ (real functions in $\mathcal{S}(\mathbf{R}))$ is left invariant by $L$ and $Y$, it follows $(L-Y)^{1 / 2} Y$ is densely defined. Thus $S$ satisfies the hypothesis of Theorem 2 , and so $S=T^{-1} i A T$ for a unitarily implementable symplectic transformation $T$ and a selfadjoint operator $A \geq \epsilon>0$. Thus

$$
\gamma_{0}(S)=\Gamma(T)^{-1} d \Gamma(A) \Gamma(T)-\langle\Gamma(T) v, d \Gamma(A) \Gamma(T) v\rangle
$$

The corollary now follows from the Corollary to Theorem 1. End.

Proof of theorem. One has first to prove existence of all the operators involved, and this is handled with the help of the following simple lemma:

Lemma. Let $A$ and $B$ be closed densely defined operators with bounded inverses. Then $A B$ is a closed densely defined operator. If $A$ and $B$ are selfadjoint, $A B A$ is selfadjoint.

Proof. $A B=\left(B^{-1} A^{-1}\right)^{-1}$ so $A B$ is closed and densely defined. In case $A$ and $B$ are selfadjoint, $A B A$ is the inverse of the bounded selfadjoint operator $A^{-1} B^{-1} A^{-1}$ and hence selfadjoint. End.

It follows $\alpha \beta^{2} \alpha$ is a selfadjoint operator, and it is obviously $\geq \epsilon^{2}$. Thus $R=\left(\alpha \beta^{2} \alpha\right)^{1 / 4} \alpha^{-1}$ is a closed densely defined operator, as $D\left(\alpha \beta^{2} \alpha\right) C$ $D\left(\left(\alpha \beta^{2} \alpha\right)^{1 / 4}\right) \cap D(\alpha)$, and $\alpha^{-1}$ is bounded. It also follows that $R^{-1}$ is closed and densely defined.

It suffices now, to prove the theorem, to show that $R^{t} R-I$ is Hilbert-Schmidt (or bounded, or trace class), as this also implies that $R$ is bounded, by the use of the polar decomposition for $R$, and that $R^{-1}$ is bounded.

But

$$
R^{t} R-I=\alpha^{-1}\left(\alpha \beta^{2} \alpha\right)^{1 / 2} \alpha^{-1}-\alpha^{-1}\left(\alpha^{4}\right)^{1 / 2} \alpha^{-1}
$$


Recalling that if $A \geq \epsilon>0$ is a selfadjoint operator, then for all $z \in D(A)$

$$
A^{1 / 2} z=\frac{1}{\pi} \int_{0}^{\infty} d y y^{-1 / 2}(y+A)^{-1} A z
$$

(Dunford-Taylor formula, see Kato [3, p. 282]), it follows that

$$
\begin{aligned}
\left(R^{t} R-I\right) z & =\frac{1}{\pi} \int_{0}^{\infty} d y y^{1 / 2} \alpha^{-1}\left(y+\alpha \beta^{2} \alpha\right)^{-1} \alpha\left(\beta^{2}-\alpha^{2}\right) \alpha\left(y+\alpha^{4}\right)^{-1} \alpha^{-1} z \\
& =\frac{1}{\pi} \int_{0}^{\infty} d y y^{1 / 2} \alpha^{-1}\left(y+\alpha \beta^{2} \alpha\right)^{-1} \alpha\left(\beta^{2}-\alpha^{2}\right)\left(y+\alpha^{4}\right)^{-1} z,
\end{aligned}
$$

for $z \in D\left(\alpha \beta^{2} \alpha \alpha^{-1}\right) \cap D\left(\alpha^{4} \alpha^{-1}\right)=D\left(\alpha\left(\beta^{2}-\alpha^{2}\right)\right)$, which is dense in $\mathcal{H}^{\prime}$ by hypothesis. As $\beta^{2}-\alpha^{2}$ is Hilbert-Schmidt (or bounded, or trace class), and $\left\|\left(y+\alpha^{4}\right)^{-1}\right\| \leq\left(y+\epsilon^{2}\right)^{-1}$, it is enough to show that $\alpha^{-1}\left(y+\alpha \beta^{2} \alpha\right)^{-1} \alpha$ is a bounded operator for $y>0$, norm continuous as a function of $y$, uniformly bounded in norm for $y$ near zero, and such that its norm is $O\left(y^{-1}\right)$ as $y \rightarrow \infty$. As $\alpha^{-1}\left(y+\alpha \beta^{2} \alpha\right)^{-1} \alpha=\left(\alpha^{-1}\left(y+\alpha \beta^{2} \alpha\right) \alpha\right)^{-1}=\left(y+\beta^{2} \alpha^{2}\right)^{-1}$, it suffices to show that $(-\infty, \delta)$ is in the resolvent set of $\beta^{2} \alpha^{2}$, for some $\delta>0$, and that $\left\|\left(y+\beta^{2} \alpha^{2}\right)^{-1}\right\|=O\left(y^{-1}\right)$ as $y \rightarrow \infty$. Let $z \in D\left(\beta^{2} \alpha^{2}\right)$, $\|z\|=1$. Then

$$
\left\langle z, \beta^{2} \alpha^{2} z\right\rangle=\left\langle z,\left(\alpha^{2}+\left(\beta^{2}-\alpha^{2}\right)\right) \alpha^{2} z\right\rangle=\left\langle z, a^{4} z\right\rangle+\left\langle z,\left(\beta^{2}-\alpha^{2}\right) z\right\rangle .
$$

But

$$
\left|\left\langle z,\left(\beta^{2}-\alpha^{2}\right) \alpha^{2} z\right\rangle\right| \leq\left\|\beta^{2}-\alpha^{2}\right\|\left\|\alpha^{2} z\right\|=\left\|\beta^{2}-\alpha^{2}\right\|\left\langle z, \alpha^{4} z\right)^{1 / 2} .
$$

Thus

$$
\begin{aligned}
\operatorname{Re}\left\langle z, \beta^{2} \alpha^{2} z\right\rangle & \geq\left\langle z, \alpha^{4} z\right\rangle-\left|\left\langle z,\left(\beta^{2}-\alpha^{2}\right) \alpha^{2} z\right\rangle\right| \\
& \geq\left\langle z, \alpha^{4} z\right\rangle-\left\|\beta^{2}-\alpha^{2}\right\|\left\langle z, \alpha^{4} z\right\rangle^{1 / 2} \geq-1 / 4\left\|\beta^{2}-a^{2}\right\|^{2},
\end{aligned}
$$

as $\left\langle z, \alpha^{4} z\right\rangle>0$. It follows it is enough to show that $\sigma\left(\beta^{2} \alpha^{2}\right) \subset\{w \in \mathbf{C} \mid \operatorname{Re} w>\delta\}$ for some $\delta>0$, where $\sigma\left(\beta^{2} \alpha^{2}\right)$ is the spectrum of $\beta^{2} \alpha^{2}$, as $\beta^{2} \alpha^{2}+1 / 4\left\|\beta^{2}-\alpha^{2}\right\|^{2}$ will then be $m$-accretive, so $\left\|\left(y+\beta^{2} \alpha^{2}\right)^{-1}\right\| \leq\left(y-1 / 4\left\|\beta^{2}-\alpha^{2}\right\|^{2}\right)^{-1}$ for $y>$ $1 / 4\left\|\beta^{2}-\alpha^{2}\right\|^{2}$ (see Kato [3, p. 279]). Actually $\sigma\left(\beta^{2} \alpha^{2}\right)$ is even nicer, in fact $\sigma\left(\beta^{2} \alpha^{2}\right) \subset\left[\epsilon^{2}, \infty\right)$, as shown by the following argument, due to W. Wils: If $\sigma^{\prime}=$ $\sigma-\{0\}$, then $\sigma^{\prime}\left(\left(\beta^{2} \alpha^{2}\right)^{-1}\right)=\sigma^{\prime}\left(\alpha^{-2} \beta^{-2}\right)=\sigma^{\prime}\left(\beta^{-1} \alpha^{-2} \beta^{-1}\right) \subset\left(0, \epsilon^{-2}\right]$, as $\sigma^{\prime}(A B)=\sigma^{\prime}(B A)$ for bounded operators $A$ and $B$, and $\alpha^{2}, \beta^{2} \geq \epsilon>0$. It follows $\sigma\left(\beta^{2} \alpha^{2}\right) \subset\left[\epsilon^{2}, \infty\right)$, as $\sigma(A)=\left(\sigma^{\prime}\left(A^{-1}\right)\right)^{-1}$ for a closed densely defined operator $A$ with a bounded inverse, as $\lambda-A=-\lambda\left(\lambda^{-1}-A^{-1}\right) A$.

To show $R^{-1}=\alpha\left(\alpha \beta^{2} \alpha\right)^{-1 / 4}$ is bounded, we repeat the argument used to show that $R$ is bounded. Using the appropriate polar decomposition for $R^{-1}$, it is enough to show that $R^{-1}\left(R^{-1}\right)^{t}$ is bounded. 
As before,

$$
R^{-1}\left(R^{-1}\right)^{t}-I=\alpha\left(\alpha \beta^{2} \alpha\right)^{-1 / 2} \alpha-\alpha\left(\alpha^{4}\right)^{-1 / 2} \alpha
$$

so

$$
\begin{aligned}
\left(R^{-1}\left(R^{-1}\right)^{t}-I\right) z & =\frac{1}{\pi} \int_{0}^{\infty} d y y^{-1 / 2} \alpha\left(y+\alpha \beta^{2} \alpha\right)^{-1} \alpha\left(\beta^{2}-\alpha^{2}\right) \alpha\left(y+\alpha^{4}\right)^{-1} \alpha z \\
& =\frac{1}{\pi} \int_{0}^{\infty} d y y^{-1 / 2}\left(\alpha^{-2} y+\beta^{2}\right)^{-1}\left(\beta^{2}-\alpha^{2}\right)\left(\alpha^{-2} y+\alpha^{2}\right)^{-1} z
\end{aligned}
$$

for $z \in D\left(\alpha\left(\alpha \beta^{2} \alpha\right)^{-1 / 2} \alpha\right)$, which is dense in $\mathcal{H}^{\prime}$. But $\alpha^{-2} y+\alpha^{2} \geq \alpha^{2} \geq \epsilon>0$, $\alpha^{-2} y+\beta^{2} \geq \beta^{2} \geq \epsilon>0$, for $y \geq 0$, so both $\left(\alpha^{-2} y+\alpha^{2}\right)^{-1}$ and $\left(\alpha^{-2} y+\beta^{2}\right)^{-1}$ are bounded operators for $y \geq 0$, norm continuous as a function of $y$, and uniformly bounded in norm for $y$ near zero. Moreover,

$$
\left(\alpha^{-2} y+a^{2}\right) \geq \inf _{\lambda \in \sigma\left(a^{2}\right)}\left(\lambda^{-2} y+\lambda^{2}\right) \geq \inf _{\lambda \in[\epsilon, \infty)}\left(\lambda^{-2} y+\lambda^{2}\right) \geq 2 y^{1 / 2}
$$

and $a^{-2} y+\beta^{2}=\alpha^{-2} y+\alpha^{2}+\left(\beta^{2}-\alpha^{2}\right) \geq 2 y^{1 / 2}-\left\|\beta^{2}-\alpha^{2}\right\|$. Thus both $\left\|\left(\alpha^{-2} y+\alpha^{2}\right)^{-1}\right\|$ and $\left\|\left(\alpha^{-2} y+\beta^{2}\right)^{-1}\right\|$ are $O\left(y^{-1 / 2}\right)$ as $y \rightarrow \infty$.

It follows $R^{-1}\left(R^{-1}\right)^{t}-I$ is a bounded operator, and thus so is $R^{-1}$.

If we let $T=R \oplus\left(R^{t}\right)^{-1}$, it is clear that $T_{S} T^{-1}=i\left(\alpha \beta^{2} \alpha\right)^{1 / 2}$. End of proof.

7. Renormalization of a quadratic Hamiltonian. Let us consider the selfinteraction of the neutral scalar free field given by the interaction Hamiltonian

$$
V=\lambda \int: \varphi(x)^{2}: d x
$$

The total Hamiltonian is then $H=H_{0}+V$. Unfortunately, $V$ cannot be defined as an operator in $\mathcal{K}$ in any reasonable sense (see Segal [10]). The problem must be reformulated. Physically, to say $H$ is the Hamiltonian means that if $\phi$ is the field, and $\dot{\phi}$ its time derivative, then $i[H, \phi(f, t)]=\dot{\phi}(f, t)$. This commutator can be computed, formally. It follows from the canonical commutation relations that $i[H, \Psi(z)]=\Psi(S z)$, for $z \in D(S)$, where $S$ is represented by the matrix

$$
\left[\begin{array}{cc}
0 & -\left(C^{2}+2 \lambda C^{-2}\right) \\
C^{2} & 0
\end{array}\right]
$$

in the decomposition $\mathcal{H}=R L_{2}\left(\mathbf{R}^{n}\right) \oplus R L_{2}\left(\mathbf{R}^{n}\right)$. If $\lambda>-m^{2} / 2$, it follows $C^{4}+$ $2 \lambda>0$, and we can define $D=\left(C^{4}+2 \lambda\right)^{1 / 4}$. Let 


$$
T=\left[\begin{array}{cc}
D^{-1} C & 0 \\
0 & D C^{-1}
\end{array}\right]
$$

Then $T$ is a bounded operator, and $\left(D^{-1} C\right)^{t} D^{-1} C=C D^{-2} C=\left(I+2 \lambda C^{-2}\right)^{-1 / 2}$, and $D^{-1} C$ is thus seen to be bounded, using its polar decomposition. $T$ is clearly a symplectic transformation, but is not unitarily implementable, as $2 \lambda C^{-2}$ is not Hilbert-Schmidt. This gives us some insight on the way $H$ cannot be defined as an operator in $K$, as

$$
T S T^{-1}=\left[\begin{array}{cc}
0 & -D^{2} \\
D^{2} & 0
\end{array}\right]=i D^{2} .
$$

It follows (see Remark 1) there exists a complex structure in $\mathcal{H}$ which makes $S$ a skew-adjoint operator. Let $\mathcal{H}_{1}$ be this new complex Hilbert space. Then $S$ is selfadjointly implementable in the corresponding free Weyl process $\left(K_{1}, W_{1}, \Gamma_{1}, v_{1}\right)$, and, as $T$ is unitary from $\mathcal{H}_{1}$ to $\mathcal{H}$, we can take $\mathcal{K}_{1}=\mathcal{K}, W_{1}=W \circ T, \Gamma_{1}(\cdot)=$ $\Gamma\left(T \cdot T^{-1}\right)$, and $v_{1}=v$. In this representation, $S$ is selfadjointly implemented by $H_{\text {ren }}=\gamma_{0}\left(T S T^{-1}\right)$. Let $S_{\text {ren }}=T S T^{-1}$. Then $S_{\text {ren }}=i D^{2}$, and thus $H_{\text {ren }}=$ $d \Gamma\left(D^{2}\right)$. Analogously to the construction of the neutral scalar free field, we define $\phi_{\text {ren }}(f)=\Psi\left(D^{-1} f\right), \dot{\phi}_{\text {ren }}(g)=\Psi(i D g)$ for real functions $f \in L_{2}\left(\mathbf{R}^{n}\right), g \in D(D)$, and we have $i\left[H_{\text {ren }}, \phi_{\text {ren }}(f)\right]^{-}=\dot{\phi}_{\text {ren }}(f)$, as desired. Thus we have been able to make sense out of $H$, but to do so we had to make a renormalization that was equivalent to a change of Hilbert space, and the need for this change came from the nonunitary implementability of $T$.

The renormalized field is just the neutral scalar free field with mass $\left(m^{2}+2 \lambda\right)^{1 / 2}$, as $D=\left(m^{2}+2 \lambda-\Delta\right)^{1 / 4}$. We have thus done a mass renormalization.

In the usual treatment, cutoffs are introduced. Their function is to make $S$ selfadjointly implementable, but they also make $T$ unitarily implementable.

The space cutoff is introduced by defining

$$
H(f)=H_{0}+\lambda \int: \varphi(x)^{2}: f(x) d x,
$$

where $f$ is a real positive function, bounded and square-integrable. This corresponds to defining

$$
S(f)=\left[\begin{array}{cc}
0 & -\left(C^{2}+2 \lambda C^{-1} M_{f} C^{-1}\right) \\
C^{2} & 0
\end{array}\right],
$$

where $M_{f}$ denotes multiplication by the function $f$. Analogously to the case 
without cutoffs, if $\lambda>-m^{2} / 2$, we define $D(f)=\left(C^{4}+2 \lambda M_{f}\right)^{1 / 4}$, and from it construct $T(f)$.

A momentum cutoff is also introduced. It substitutes $M_{f, \sigma}$ for $M_{f}$, where the operator $M_{f, \sigma}$ is defined as having, in Fourier transform space, the integral kernel $X_{\sigma}(k) \hat{f}(k-p) X_{\sigma}(p)$, where $X_{\sigma}(k)=1$ if $k \leq \sigma$, and is zero otherwise. As this is an $L_{2}$-kernel, $M_{f, \sigma}$ is a Hilbert-Schmidt operator. Thus, $S(f, \sigma)=$ $i\left(C^{2}+\lambda C^{-1} M_{f, \sigma} C^{-1}\right)+K(f, \sigma)$, where

$$
K(f, \sigma)=\left[\begin{array}{cc}
0 & -\lambda C^{-1} M_{f, \sigma} C^{-1} \\
-\lambda C^{-1} M_{f, \sigma} C^{-1} & 0
\end{array}\right]
$$

is a self-transpose Hilbert-Schmidt infinitesimal symplectic. It follows $S(f, \sigma)$ is selfadjointly implementable, and we can set $H(f, \sigma)=\gamma_{0}(S(f, \sigma))$. On the other hand, $T(f, \sigma)$ is unitarily implementable, as $C D(f, \sigma)^{-2} C=\left(C^{-1} D(f, \sigma)^{2} C^{-1}\right)^{-1}=$ $\left(I+C^{-1}\left(D(f, \sigma)^{2}-C^{2}\right) C^{-1}\right)^{-1}$, and $D(f, \sigma)^{2}-C^{2}$ is seen to be Hilbert-Schmidt using the Dunford-Taylor formula, as in the proof of Theorem 2 (only here it is very easy to see that the integrand is bounded, with Hilbert-Schmidt norm bounded by an integrable function), and it is easy to see that if $X$ is a Hilbert-Schmidt operator, and $I+X$ is invertible, then $(I+X)^{-1}=I+Y$, where $Y$ is again Hilbert-Schmidt. It follows there exists a constant $E(f, \sigma)$ such that $\Gamma(T(f, \sigma))$. $(H(f, \sigma)+E(f, \sigma)) \Gamma(T(f, \sigma))^{-1}=\gamma_{0}\left(T(f, \sigma) S(f, \sigma) T(f, \sigma)^{-1}\right)=H(f, \sigma)_{\text {ren }}$, as both sides generate continuous unitary one-parameter groups implementing the same continuous one-parameter group of symplectic transformations. The constant $E(f, \sigma)$ is needed to adjust the ground state energy of $H(f, \sigma)$ to zero.

Similarly, the techniques of Theorem 2 also show that $C^{-1}\left(D^{2}(f)-C^{2}\right) C^{-1}$ is Hilbert-Schmidt, if the number of space dimensions is $\leq 3$, so $T(f)$ is unitarily implementable, and it follows that $S(f)$ is selfadjointly implementable, and we can repeat the above procedure with $S(f)$. But $K(f)$ is not Hilbert-Schmidt (see Remark 2).

To remove the cutoffs, we make first $\sigma \rightarrow \infty$, and then $f \rightarrow 1$. It is easy to see that $D^{4}(f, \sigma) \rightarrow D^{4}(f)$ strongly in the domain of $D^{4}(f)$, as $\sigma \rightarrow \infty$. As $D^{4}(f, \sigma), D^{4}(f)$ are uniformly bounded away from zero, we can use the DunfordTaylor formula to get $D^{2}(f, \sigma) \rightarrow D^{2}(f)$ strongly in the domain of $D^{2}(f)$, as $\sigma \rightarrow \infty$. By a theorem of Trötter, $e^{t S(f, \sigma)}$ ren $\rightarrow e^{t S(f)^{2}}$ strongly, so it follows $e^{i t H(f, \sigma)_{\text {ren }}} \rightarrow e^{i t H(f)_{\text {ren }}}$. Similarly, $e^{t S(f)_{\text {ren }}} \rightarrow e^{t S_{\text {ren }}}$ strongly, and
$e^{i t H(f)_{\text {ren }}} \rightarrow e^{i t H_{\text {ren }}}$ strongly. 


\section{REFERENCES}

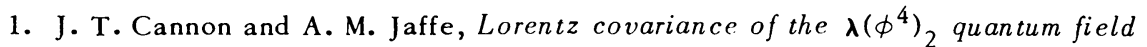
theory, Comm. Math. Phys. 17 (1970), 261-321. MR 42 \#182.

2. J. Ginibre and G. Velo, Renormalization of a quadratic interaction in the Hamiltonian formalism, Comm. Math. Phys. 18 (1970), 65-81. MR 42 \#1436.

3. T. Kato, Perturbation theory for linear operators, Die Grundlehren der math. Wissenschaften, Band 132, Springer-Verlag, New York, 1966. MR 34 \#3324.

4. N. Skovhus Poulsen, Private communication (preprint in preparation).

5. L. Rosen, The $\left(\phi^{2 n}\right)_{2}$ quantum field theory: Lorentz covariance, J. Math. Anal. Appl. 38 (1972), 276-311.

6. - Renormalization of the Hilbert space in the mass shift model, J. Mathematical Phys. 13 (1972), 918-927.

7. I. Segal, Tensor algebras over Hilbert spaces. I, Trans. Amer. Math. Soc. 81 (1956), 106-134. MR 17, 880.

8. - Conjugacy to unitary groups within the infinite dimensional symplectic group, Argonne National Laboratory report ANL-7216, 1966.

9. - Nonlinear functions of weak processes. II, J. Functional Analysis 6 (1970), 29-75. MR $41 \# 7974$.

10. - Transformations in Wiener space and squares of quantum fields, Advances in Math. 4 (1970), 91-108. MR $42 \# 8826$.

11. - Construction of non-linear local quantum processes. I, Ann. of Math. (2) 92 (1970), 462-481; Erratum, ibid. (2) 93 (1971), 597. MR $42 \# 7187$.

12. Construction of nonlinear local quantum processes. II, Invent. Math. 14 (1971), 211-241.

13. D. Shale, Linear symmetries of free Boson fields, Trans. Amer. Math. Soc. 103 (1962), 149-167. MR 25 \#956.

14. M. Weinless, Existence and uniqueness of the vacuum for linear quantized fields, J. Functional Analysis 4 (1969), 350-379. MR 40 \#6901.

DEPARTMENT OF MATHEMATICS, UNIVERSITY OF CALIFORNIA, LOS ANGELES, CALIFORNIA 90024

Current address: Department of Mathematics, Princeton University, Princeton, New Jersey 08540 